\title{
Pacific
}

Journal of

Mathematics

\section{LIPSCHITZ AND BILIPSCHITZ MAPS ON CARNOT GROUPS}

WILLIAM MEYERSON

Volume 263 No. 1

May 2013 


\title{
LIPSCHITZ AND BILIPSCHITZ MAPS ON CARNOT GROUPS
}

\author{
WILLIAM MEYERSON
}

\begin{abstract}
Suppose $A$ is an open subset of a Carnot group $G$ and $H$ is another Carnot group. We show that a Lipschitz function from $A$ to $H$ whose image has positive Hausdorff measure in the appropriate dimension is bilipschitz on a subset of $A$ of positive Hausdorff measure. We also construct Lipschitz maps from open sets in Carnot groups to Euclidean space that do not decrease dimension. Finally, we discuss two counterexamples to explain why Carnot group structure is necessary for these results.
\end{abstract}

\section{Introduction}

Guy David [1988] proved that if $f$ is a Lipschitz function from the unit cube in $\mathbb{R}^{n}$ to a subset of some Euclidean space with positive $n$-dimensional Hausdorff measure, there exists a subset $K$ of the domain of $f$ with positive $n$-dimensional Hausdorff measure such that $f$ is bilipschitz on $K$.

Shortly thereafter, Peter Jones [1988] proved the following stronger result: if $f$ is a Lipschitz function from the unit cube in $\mathbb{R}^{n}$ to a subset of some Euclidean space, then the unit cube can be broken up into the union of a "garbage" set (whose image under $f$ has arbitrarily small $n$-dimensional Hausdorff content) and a finite number of sets $K_{1}, \ldots, K_{N}$ such that $f$ is bilipschitz on each $K_{i}$.

David [1991] later translated this proof into the language of wavelets, which are more readily generalizable to Heisenberg and other Carnot groups. The proof as written in [David 1991] only depends on a few general properties, all but one of which hold for Heisenberg (and other Carnot) groups.

This story has further generalizations: for example, [David and Semmes 1993] generalizes Jones' argument to work with Lipschitz functions that are only defined on Ahlfors $d$-regular subsets of a Euclidean space $\mathbb{R}^{N}$, with $d$ possibly less than $N$, while [Semmes 2000] allows the domain and range to be metric spaces subject to a specific condition.

In Section 2 we adapt some of the ideas in [David 1991; Jones 1988] to Carnot groups and prove that a Lipschitz function between such groups having an image of positive Hausdorff measure in the appropriate dimension is bilipschitz on a subset

Keywords: analysis on Carnot groups, Heisenberg groups, Grushin plane, subriemannian, wavelets. 
of the domain of positive Hausdorff measure. Section 3 investigates how big, in terms of dimension, Lipschitz images of Carnot groups in Euclidean space can be. Finally, Section 4 explores two counterexamples explaining why Carnot group structure is necessary for these results. In particular, neither Ahlfors regularity nor subriemannian manifold structure would be sufficient.

\section{Jones-type decomposition for Carnot groups}

2A. Brief outline. This section is organized as follows. In Section $2 \mathrm{~B}$ we give some definitions concerning Carnot groups and set up some notational conventions. In Section 2C we state the five properties of Euclidean space on which David's argument rests and show how the first four of them work for Heisenberg groups. In Section 2D we explain why these properties also work for other Carnot groups. In Section 2E, we prove our main result (Theorem 2.12): If $A$ is an appropriate subset of the $k$-th Heisenberg group $H_{k}$ corresponding roughly to the unit cube in $\mathbb{R}^{n}$, and $F$ is a Lipschitz function from $A$ to another Heisenberg group whose image has positive Hausdorff $(2 k+2)$-dimensional measure, then there exists $B \subset A$ with positive Hausdorff $(2 k+2)$-dimensional measure such that $F$ is bilipschitz on $B$. Finally, in Section 2F we derive some corollaries of Theorem 2.12.

Although our main focus is on the Heisenberg groups (especially $H_{1}$ ), all of the results in this paper apply equally well to Carnot groups in general. To exploit this fact, the results in Section $2 \mathrm{E}$ will be stated and proved in the more general context of Carnot groups.

\section{B. Definitions.}

Definition 2.1. The $n$-th Heisenberg group $H_{n}$ is defined as the set

$$
\left\{\left(z_{1}, \ldots, z_{n}, t\right): z_{j} \in \mathbb{C}, t \in \mathbb{R}\right\}
$$

equipped with the following group law:

$$
\left(z_{1}, \ldots, z_{n}, t\right)\left(w_{1}, \ldots, w_{n}, s\right)=\left(z_{1}+w_{1}, \ldots, z_{n}+w_{n}, t+s+\Im \sum_{j=1}^{n} z_{j} \bar{w}_{j}\right),
$$

where $\mathfrak{s}$ denotes imaginary part.

For $n=1$, we often write $z_{1}$ in terms of its real components as $z_{1}=x+i y$ and refer to the point $\left(z_{1}, t\right)$ as $(x, y, t)$, so $H_{1}$ inherits a natural Euclidean coordinate structure from $\mathbb{R}^{3}$.

The Heisenberg group is a special example of a Carnot group:

Definition 2.2. A Carnot group $G$ is a connected, simply connected, nilpotent Lie group whose Lie algebra $\mathfrak{g}$ is graded, i.e.,

$$
\mathfrak{g}=\bigoplus_{j=1}^{d} \mathfrak{g}_{j}
$$


where

$$
\left[\mathfrak{g}_{1}, \mathfrak{g}_{j}\right]=\mathfrak{g}_{j+1} \quad \text { and } \quad \mathfrak{g}_{d+1}=\{0\} .
$$

We call $\mathfrak{g}_{1}$ the horizontal component of $\mathfrak{g}$.

By standard results of Lie group theory (see, for example, [Varadarajan 1984]), the exponential map gives a diffeomorphism between a Carnot group and its Lie algebra. Further, the standard definition of a Lie algebra in terms of vector fields provides a canonical identification between the tangent space of a Lie group at a given point and the Lie group itself. (When $g \in G$ is fixed, for every tangent vector $v$ there is a unique $X \in \mathfrak{g}$ such that $X(g)=v$ and we can identify $\exp (X)$ with $v$.)

We shall freely use these canonical identifications between a Carnot group, its Lie algebra, and its tangent space throughout this paper. For example, every Carnot group has a coordinate structure induced by its Lie algebra. For $H_{n}$, this coordinate structure was already mentioned in Definition 2.1, where $\mathfrak{g}_{1}$ consists of the points of the form $\left(z_{1}, \ldots, z_{n}, 0\right)$ with final coordinate equal to zero.

Every Carnot group has a family of dilation homomorphisms $\left\{\delta_{\lambda}: \lambda>0\right\}$ and a metric called the Carnot-Carathéodory metric. They are defined as follows:

Definition 2.3. Let $\lambda>0$, let $G$ be a Carnot group and let $g \in G$, where $g=\sum_{i} g_{i}$ with $g_{i} \in \mathfrak{g}_{i}$. Define the dilation

$$
\delta_{\lambda}(g)=\sum_{i} \lambda^{i} g_{i}
$$

Definition 2.4. Let $G$ be a Carnot group, let $g, h \in G$, and let $\Gamma_{g, h}$ be the set of all curves

$$
\gamma:[0,1] \rightarrow G
$$

with $\gamma(0)=g, \gamma(1)=h$, and $\gamma^{\prime}(t) \in \mathfrak{g}_{1}$ for each $t \in[0,1]$. Define the CarnotCarathéodory distance between $g$ and $h$ to be

$$
d_{C C}(g, h)=\inf _{\gamma \in \Gamma_{g, h}} \int_{0}^{1}\left|\gamma^{\prime}(t)\right| d t,
$$

where $\left|\gamma^{\prime}(t)\right|$ is the length of $\gamma^{\prime}(t)$ in a fixed Euclidean metric on the real vector space $\mathfrak{g}_{1}$.

Because $\Gamma_{g, h}$ in this definition is nonempty — see [Montgomery 2002] — we have $d_{C C}(g, h)<\infty$ whenever $g, h \in G$.

Note 2.5. It is often easier to work with a comparable $L^{\infty}$ quasidistance function $d$ based on the Carnot metric. For the first Heisenberg group $H_{1}$, this is done by defining distance to the origin as

$$
d((x, y, z),(0,0,0))=\max \left(|x|,|y|,|z|^{1 / 2}\right)
$$

and for an arbitrary $g, h$ in this group, defining 


$$
d(g, h)=d\left(h^{-1} g,(0,0,0)\right) .
$$

There is of course a completely analogous construction in an arbitrary Carnot group: if $G$ is a Carnot group, we use the grading of its Lie algebra $\mathfrak{g}$ as in the definition of Carnot groups:

$$
\mathfrak{g}=\bigoplus_{j=1}^{d} \mathfrak{g}_{j}
$$

Because the identity element in a Carnot group is the image of the origin under the exponential map, we shall refer to it as 0 . Now, letting $g$ be an arbitrary point in $G$ we first define its quasidistance to the identity element, $d(g, 0)$, by recalling the direct sum decomposition

$$
\exp ^{-1}(g)=\sum_{j} g_{j}
$$

with $g_{j} \in \mathfrak{g}_{j}$ and setting

$$
d(g, 0)=\max _{1 \leq j \leq d}\left(\left\|g_{j}\right\|_{j}\right)^{1 / j} .
$$

where $\|\cdot\|_{j}$ is a norm on $\mathfrak{g}_{j}$ for $j=1, \ldots, d$. Finally, for an arbitrary $g, h \in G$, we finish by setting

$$
d(g, h)=d\left(h^{-1} g, 0\right) .
$$

For the duration of this paper, $d_{C C}$ shall refer to Carnot-Carathéodory distance and $d$ shall refer to quasidistance.

A fundamental operation for Carnot groups is the Pansu differential, defined as follows (see [Capogna et al. 2007], for example):

Definition 2.6. Let $F: G \rightarrow H$ be a function from one Carnot group $G$ to another Carnot group $H$. The Pansu differential $D F(g)$ of $F$ at $g \in G$ is the map

$$
D F(g): G \rightarrow H
$$

defined at $g^{\prime} \in G$ as the limit

$$
D F(g)\left(g^{\prime}\right)=\lim _{s \rightarrow 0} \delta_{s^{-1}}\left[F(g)^{-1} F\left(g \delta_{s} g^{\prime}\right)\right]
$$

whenever the limit exists.

Using the canonical identifications stated above, we can view the Pansu differential as a map between Lie algebras or as a map from the tangent space at $g \in G$ to the tangent space at $F(g)$. We shall take advantage of this fact throughout.

In the tangent vector interpretation, the Pansu differential $D F(g)$ induces a linear map between the horizontal component of the tangent space of $G$ at $g$ and the horizontal component of the tangent space of $H$ at $F(g)$ [Pansu 1989]. Calling this linear map $M F(g)$, we can view $M F$ as a matrix-valued map sending $g$ to $M F(g)$. 


\section{C. Five key properties.}

2C1. Dyadic decomposition. There exists a dyadic decomposition for Euclidean space defined as follows: For each nonnegative integer $k$ we let $2_{k}$ be the set of all cubes of the form

$$
\left(a_{1} \cdot 2^{-k},\left(a_{1}+1\right) \cdot 2^{-k}\right) \times \cdots \times\left(a_{n} \cdot 2^{-k},\left(a_{n}+1\right) \cdot 2^{-k}\right)
$$

contained in the unit cube, where the $a_{i}$ are all integers. Then the elements of $2_{k}$ are disjoint open sets. Further, each element of $2_{k}$ is (up to a set of measure zero) a disjoint union of elements of $2_{k+1}$, the $2_{k}$ are all translates of each other, and one can transform an arbitrary element of $2_{k}$ into an arbitrary element of $2_{k+1}$ by a dilation (by a factor of $2^{-1}$ ) followed by translation. Finally, fixing a cube $Q \in 2_{k}$ and letting $d$ be its diameter (i.e., $d=\sqrt{n} 2^{-k}$ ), the number of cubes in $2_{k}$ whose distance from $Q$ is at most $d$ is bounded above by a constant depending only on $n$.

Our immediate goal is to generalize this decomposition to the Heisenberg group $H_{1}$. To do this we loosely follow Christ's construction of Theorem 11 in [Christ 1990]. First we let $B_{0}$ denote the discrete subgroup of $H_{1}$ generated by $(1,0,0)$ and $(0,1,0)$ and call it the discrete Heisenberg group. We then define $B_{n}$, for each positive integer $n$, to be the image of $B_{0}$ under the dilation $\delta_{10^{-n}}$ (in particular, the first 2 coordinates are multiplied by $10^{-n}$; the final coordinate is multiplied by $\left.10^{-2 n}\right)$. Equivalently, $B_{n}$ is the subgroup of the first Heisenberg group generated by $\left(10^{-n}, 0,0\right)$ and $\left(0,10^{-n}, 0\right)$. If $x$ is a point in $B_{n}$, we give it the label $(x, n)$ and note that $x$ has a different label for each $B_{n}$ containing $x$. We form a tree by defining an order relation $\leq$ on the set of all such pairs $(x, n)$. We start this procedure with the following definition.

Definition 2.7. We say that $(x, \alpha)$ is a parent of $(y, \beta)$ if $\beta=\alpha+1$ and $y=x g$, where the first two components of $g$ all lie in $\left(-\frac{1}{2} 10^{-\alpha}, \frac{1}{2} 10^{-\alpha}\right]$ and the final component lies in $\left(-\frac{1}{2} \cdot 10^{-2 \alpha}, \frac{1}{2} \cdot 10^{-2 \alpha}\right]$.

Using the obvious analogies from family trees ("ancestor", "descendant", "grandparent", "sibling", etc.) for both the tree points and corresponding dyadic cubes (to be defined momentarily), we say $(x, \alpha) \leq(y, \beta)$ if $(y, \beta)$ is an ancestor of $(x, \alpha)$. Following along exactly as in Definition 14 of [Christ 1990], we create from this tree a family of dyadic "cubes". In particular, we define

$$
Q(x, \alpha)=\bigcup_{(y, \beta) \leq(x, \alpha)} B_{C C}\left(y, \frac{1}{10} 10^{-\beta}\right),
$$

where $B_{C C}(z, \epsilon)$ is the ball centered at $z$ of radius $\epsilon$ with respect to CarnotCarathéodory distance. We will say that each cube $Q(x, \alpha)$ is a cube at scale $\alpha$ and we define $2_{\alpha}$ to be the set of all the cubes of scale $\alpha$. All the cubes in $2_{\alpha}$ are translates of each other by elements of the discrete Heisenberg group of 
the appropriate scale; further, each member of each $2_{\alpha}$ is an open set while each element of $2_{\alpha}$ is (up to a set of measure zero) the disjoint union of elements of $2_{\alpha+1}$. Also, one can transform an arbitrary element of $2_{\alpha}$ into an arbitrary element of $2_{\alpha+1}$ by a dilation (by a factor of $10^{-1}$ ) followed by translation. Finally, the number of cubes in $2_{\alpha}$ within $\operatorname{diam}(Q(x, \alpha))$ of $Q(x, \alpha)$ is bounded by a constant independent of $\alpha$.

Analogously, for the $k$-th Heisenberg group, we begin by rewriting the elements of $H_{k}$ to mirror the above construction for $H_{1}$ : in other words, writing $z_{j}=x_{2 j-1}+i x_{2 j}$ where $x_{2 j-1}, x_{2 j} \in \mathbb{R}$, we let $B_{0}$ be the subgroup of $H_{k}$ generated by

$$
\left\{\left(x_{1}, \ldots, x_{2 k}, 0\right): x_{j}= \pm \delta_{j, l}, 1 \leq l \leq 2 k\right\}
$$

where $\delta_{j, l}$ is the Kronecker delta. In this setting, $B_{n}$ would be the subgroup of $H_{k}$ generated by

$$
\left\{\left(x_{1}, \ldots, x_{2 k}, 0\right): x_{j}= \pm 10^{-n} \delta_{j, l}, 1 \leq l \leq 2 k\right\}
$$

and the construction for $H_{1}$ goes through for $H_{k}$ with only minor changes. In particular, the definition of $(x, \alpha)$ being a parent of $(y, \beta)$ would now require $y=x g$ where the first $2 k$ components of $g$ all lie in $\left(-\frac{1}{2} 10^{-\alpha}, \frac{1}{2} 10^{-\alpha}\right]$ and the final component lies in $\left(-\frac{1}{2} \cdot 10^{-2 \alpha}, \frac{1}{2} \cdot 10^{-2 \alpha}\right]$.

In this construction, the analogue to the unit cube in Euclidean space is the unique cube of scale 0 containing the identity element; according to the notation defined in the preceding paragraph, the name for this cube is $Q(0,0)$.

Remark. In making this decomposition we are saying nothing about the boundaries of the elements of the $2_{\alpha}$ other than that they are closed sets of Hausdorff measure zero in the appropriate dimension. Also, this decomposition is not the same as the decomposition of the Heisenberg group found in [Strichartz 1992].

2C2. Orthogonal decomposition of $L^{2}$. Looking back at Euclidean space $\mathbb{R}^{n}$ for inspiration, we note that the Hilbert space $L^{2}\left([0,1]^{n}\right)$ of square-integrable functions on the unit cube can be decomposed into orthogonal subspaces as follows: if $\beta$ is a positive integer, we define $C_{\beta} \subset L^{2}\left([0,1]^{n}\right)$ as

$$
\left\{f \in L^{2}\left([0,1]^{n}\right):\left.f\right|_{Q} \text { is constant for } Q \in \mathscr{2}_{\beta} \text { and } \int_{Q} f=0 \text { for } Q \in \mathscr{2}_{\beta-1}\right\},
$$

while $C_{0} \subset L^{2}\left([0,1]^{n}\right)$ is defined as

$$
\left\{f \in L^{2}\left([0,1]^{n}\right): f \text { is constant }\right\} .
$$

This yields the orthogonal decomposition

$$
L^{2}\left([0,1]^{n}\right)=\bigoplus_{\beta=0}^{\infty} C_{\beta} .
$$


In other words, if $f \in C_{\beta}, g \in C_{\gamma}$ with $\beta \neq \gamma, \int_{[0,1]^{n}} f g=0$ while for each $h \in L^{2}\left([0,1]^{n}\right)$ there exists $h_{\beta} \in C_{\beta}$ for $\beta$ a nonnegative integer with $h=\sum_{\beta=0}^{\infty} h_{\beta}$, the sum in question converging in $L^{2}\left([0,1]^{n}\right)$ to $h$.

For the Heisenberg groups we can mimic this procedure as follows: here, our "base" cube shall be denoted as $Q(0,0)$ where the first zero denotes the origin and the second zero denotes scale. Similarly, we define the $C_{\beta}$ (as subspaces of the Hilbert space $L^{2}(Q(0,0))$ of real-valued, square-integrable functions) identically to the way we did with Euclidean space. In other words, if $\beta$ is a positive integer, we define $C_{\beta} \subset L^{2}(Q(0,0))$ as

$$
\left\{f \in L^{2}(Q(0,0)):\left.f\right|_{Q} \text { is constant for } Q \in \mathscr{2}_{\beta} \text { and } \int_{Q} f=0 \text { for } Q \in \mathscr{2}_{\beta-1}\right\} \text {, }
$$

while $C_{0} \subset L^{2}(Q(0,0))$ is defined as

$$
\left\{f \in L^{2}(Q(0,0)): f \text { is constant }\right\} .
$$

This yields the orthogonal decomposition

$$
L^{2}(Q(0,0))=\bigoplus_{\beta=0}^{\infty} C_{\beta}
$$

For $\beta>0, C_{\beta}$ has a spanning set consisting of the functions $f_{Q, Q^{\prime}}$ for $Q, Q^{\prime}$ sibling cubes in $2_{\beta}$ defined as follows: $f_{Q, Q^{\prime}}$ is equal to 1 on $Q,-1$ on $Q^{\prime}$, and 0 everywhere else; we shall call this spanning set $S_{\beta} . S_{\beta}$ is approximately orthogonal in the following sense: there exists some universal constant $K$ (independent of $\beta$ ) such that for each $f \in S_{\beta}$ we have

$$
\#\left\{g \in S_{\beta}: \int_{Q(0,0)} f g \neq 0\right\} \leq K,
$$

where the \# symbol denotes cardinality.

When we proceed to the proof, we will wish to find a fixed $Y>0$ such that if $g, g^{\prime} \in Q(0,0)$ with $g \neq g^{\prime}$, there exists some dyadic cube $Q$ such that

$$
\operatorname{diam}(Q)<Y d_{C C}\left(g, g^{\prime}\right) \text { and } g, g^{\prime} \in Q .
$$

This is arranged by considering not just the cube families $2_{\alpha}$ discussed in the previous section but expanding each cube family $\mathscr{2}_{\alpha}$ to a larger family $\mathscr{2}_{\alpha}^{\prime}$.

If $\alpha>0$, we define $2_{\alpha}^{\prime}$ to consist of the cubes of the form

$$
\left\{g Q: Q \in 2_{\alpha}, g \in B_{\alpha+2}\right\} ;
$$

remember that $B_{\alpha+2}$ was defined in the previous subsection as the discrete Heisenberg group of scale $\alpha+2$.

This does not cause the number of dyadic cubes of a given scale to multiply unreasonably because writing $g \in B_{\alpha+2}$ in coordinate form as $\left(z_{1}, \ldots, z_{n}, t\right)$, every 
element of $2_{\alpha}^{\prime}$ can be written as $g Q$ for some $Q \in 2_{\alpha}$ and $g \in B_{\alpha+2}$ with

$$
z_{1}, \ldots, z_{n} \in\left[-10^{-\alpha}, 10^{-\alpha}\right] \text { and } t \in\left[-10^{-2 \alpha}, 10^{-2 \alpha}\right] .
$$

Letting $L_{g}$ denote left translation by $g$ whenever $g \in H^{k}$, we then define

$$
C_{\beta}^{\prime}=\left\{f \circ L_{g^{-1}}: f \in C_{\beta}, g \in B_{\beta+2}\right\} .
$$

In fact, writing $g \in B_{\beta+2}$ in coordinate form as $\left(z_{1}, \ldots, z_{n}, t\right)$, every element of $C_{\beta}^{\prime}$ can be written as $f \circ L_{g^{-1}}$ for some $f \in C_{\beta}$ and $g \in B_{\beta+2}$ with

$$
z_{1}, \ldots, z_{n} \in\left[-10^{-\beta}, 10^{-\beta}\right] \text { and } t \in\left[-10^{-2 \beta}, 10^{-2 \beta}\right]
$$

Fixing $\beta$, we can construct an approximately orthogonal basis for $C_{\beta}^{\prime}$ analogously to the way we did for each $C_{\beta}$ : we simply construct an approximately orthogonal basis for $C_{\beta} \circ L_{g^{-1}}$ for each $g$ separately.

Finally, for both Euclidean space and the Heisenberg group, it is occasionally necessary to work with sets on a slightly larger scale than the unit cube. To do this, one fixes some integer $k \leq 0$, denotes our base cube to be the cube of scale $k$ which contains $Q(0,0)$, and then defines $C_{\beta}$ and $C_{\beta}^{\prime}$ appropriately for $\beta \leq 0$ (for example, $C_{k}$ will consist of the constant functions on our new base cube here).

2C3. Differentiability. On the Euclidean unit cube, there exists a Jacobian map that sends each Lipschitz function $f$ (which may be either scalar-valued or Euclidean vector-valued) on the unit cube to the almost-everywhere-defined function $J f$, the Jacobian of $f$. At almost every point, the Jacobian is a linear map from the tangent space of the domain to the tangent space of the image. Further, the partial derivative of each component is bounded above by the Lipschitz coefficient of $f$. Finally, a Lipschitz function $f$ with almost everywhere constant Jacobian defined on a connected open set is uniquely determined by this Jacobian and its value at a single point: if $J f$ is equal to the linear map $T$ almost everywhere and $f\left(x_{0}\right)=y_{0}$ then

$$
f(x)=T\left(x-x_{0}\right)+y_{0} \text { for all } x \text { where } f(x) \text { is defined. }
$$

Similarly, if $G$ and $H$ are two Heisenberg groups and $F: G \rightarrow H$ is Lipschitz, then by [Pansu 1989] the Pansu differential $D F$ (which, for almost every $g \in G$ induces a map $D F(g): G \rightarrow H)$ satisfies these three properties:

(i) At almost every $g \in G$, the differential of the Lie group map $D F(g)$ at the identity induces a Lie algebra homomorphism from the tangent space of $G$ at $g$ to the tangent space of $H$ at $F(g)$.

(ii) The magnitude of each component of $D F$ is bounded above (up to a constant depending on normalization) by the Lipschitz coefficient of $F$. 
(iii) If for almost every $g$ with respect to Haar measure on $G, D F(g)$ (which was defined as an $H$-valued function defined on $G$ ) is equal to the Lie group homomorphism $\phi: G \rightarrow H$ and $g_{0} \in G, h_{0} \in H$ with $F\left(g_{0}\right)=h_{0}$ then

$$
F(g)=h_{0} \phi\left(g_{0}^{-1} g\right) \quad \text { for all } g \text { where } F(g) \text { is defined. }
$$

Of the properties, only (iii) is not a simple consequence of [Pansu 1989]. However, (iii) is a direct consequence of this fact concerning uniqueness of Lipschitz maps:

Fact 2.8. Suppose $G$ and $H$ are Carnot groups, $U \subset G$ is connected and open, $g_{0} \in U$ and $F_{1}: U \rightarrow G$ and $F_{2}: U \rightarrow G$ are two Lipschitz maps such that $D F_{1}(g)=$ $D F_{2}(g)$ for almost all $g \in U$ with respect to Haar measure and $F_{1}\left(g_{0}\right)=F_{2}\left(g_{0}\right)$. Then $F_{1}=F_{2}$.

Proof. Suppose there exists $u \in U$ with $F_{1}(u) \neq F_{2}(u)$. Fix $\epsilon>0$ such that

$$
d_{C C}\left(F_{1}(u), F_{2}(u)\right)>\epsilon .
$$

Let $\gamma$ be a piecewise horizontal curve in $U$ joining $g_{0}$ to $u$. There exists $g^{\prime} \in G$ sufficiently close to the identity such that the left translation of $\gamma$ by $g^{\prime}$ lies in $U$ (which implies that $\left.g^{\prime} g_{0}, g^{\prime} u \in U\right)$ with $d_{C C}\left(F_{1}\left(g^{\prime} g_{0}\right), F_{2}\left(g^{\prime} u\right)\right)>\epsilon$ and almost everywhere on this translation, $D F_{1}=D F_{2}$. However, integration then implies

$$
F_{1}\left(g^{\prime} u\right) F_{1}\left(g^{\prime} g_{0}\right)^{-1}=F_{2}\left(g^{\prime} u\right) F_{2}\left(g^{\prime} g_{0}\right)^{-1} .
$$

Therefore, we know that $d_{C C}\left(F_{1}\left(g^{\prime} u\right), F_{2}\left(g^{\prime} u\right)\right)=d_{C C}\left(F_{1}\left(g^{\prime} g_{0}\right), F_{2}\left(g^{\prime} g_{0}\right)\right)>\epsilon$; since $g^{\prime}$ can be made arbitrarily close to the identity this gives us that

$$
\epsilon \leq d_{C C}\left(F_{1}(u), F_{2}(u)\right)=d_{C C}\left(F_{1}\left(g_{0}\right), F_{2}\left(g_{0}\right)\right)=0
$$

producing a contradiction, so we conclude that $F_{1}=F_{2}$ as desired.

In fact, because each linear map $\psi$ from the horizontal component of $G$ to the horizontal component of $H$ has at most one extension (which we call $\tilde{\psi}$ ) to a Lie group homomorphism from $G$ to $H$, we can go one step further and say that if $M F$ is equal to the linear map $\psi$ almost everywhere and $g_{0} \in G, h_{0} \in H$ with $F\left(g_{0}\right)=h_{0}$ then

$$
F(g)=h_{0} \tilde{\psi}\left(g_{0}^{-1} g\right)
$$

for all $g$ where $F(g)$ is defined.

2C4. Weak convergence. If a sequence $f_{n}$ of uniformly Lipschitz functions on a bounded Euclidean region converges uniformly to some function $f$ then $f$ is Lipschitz, and moreover the Jacobians $J f_{n}$ converge weakly in $L^{2}$ to the Jacobian of $f$. In other words: 
Fact 2.9. Let $U \subset \mathbb{R}^{k}$ be a bounded open set and let $\left\{f_{n}\right\}: U \rightarrow \mathbb{R}^{m}$ be a sequence of uniformly Lipschitz functions which converges uniformly to the function $f: U \rightarrow \mathbb{R}^{m}$. If $g: U \rightarrow \mathbb{R}$ is an $L^{2}$ function and $D$ represents partial differentiation with respect to a fixed vector in $\mathbb{R}^{k}$ then

$$
\int_{U}\left(D f_{n}\right) g \rightarrow \int_{U}(D f) g
$$

where the integrals are with respect to Lebesgue measure and the derivatives in question are defined almost everywhere.

As will be stated shortly, Fact 2.9 generalizes to Heisenberg groups when the map $M F$ induced by the Pansu differential (see the definitions section) is used in place of the Jacobian. In particular, one notes that because $M F$ consists of derivatives of horizontal components of $F$ with respect to horizontal tangent vectors, $M F$ can be viewed as an array of horizontal derivatives of real-valued Lipschitz functions (after postcomposing with the appropriate coordinate functions). Then, the weak convergence in question is the following fact:

Fact 2.10. Let $U \subset H_{k}$ be a bounded open set and let $\left\{f_{n}\right\}: U \rightarrow H_{m}$ be a sequence of uniformly Lipschitz functions which converges uniformly to the function $f: U \rightarrow H_{m}$. If $g: U \rightarrow \mathbb{R}$ is an $L^{2}$ function (with respect to Haar measure) and $D$ represents partial differentiation with respect to a fixed left-invariant horizontal vector field in $H_{k}$ then

$$
\int_{U}\left(D f_{n}\right) g \rightarrow \int_{U}(D f) g
$$

where the integrals are with respect to Haar measure and the derivatives in question are defined almost everywhere.

Facts 2.9 and 2.10 have the same classical proof, which involves approximating $g$ by sufficiently smooth test functions with compact support and integrating by parts.

2C5. Lipschitz extension. If $A$ is a subset of the unit cube of $\mathbb{R}^{n}$ and $f$ is a Lipschitz function from $A$ to some Euclidean space, then $f$ can be extended to a Lipschitz function on the entire unit cube (or, in fact, to all of $\mathbb{R}^{n}$ for that matter). It is not known whether this extension property also holds for maps from a subset of a Heisenberg group $G$ into the same group $G$ [Balogh and Fässler 2009; Brudnyi and Brudnyi 2007], and for that reason we assume the Lipschitz map in Corollary 2.17 below is defined on an open subset of $G$. It has been shown recently in [Balogh and Fässler 2009] that this extension property does not hold for maps from $\mathbb{R}^{k}$ to $H_{n}$ with $n<k$. Also, [Rigot and Wenger 2010] shows that the property does not hold for maps from $\mathbb{R}^{k}$ to any jet space on $H_{n}$ whenever $n<k$. However, this property does hold for maps from any Carnot group to any $\mathbb{R}^{k}$. It also holds for maps from $\mathbb{R}^{2}$ to $H_{n}$ for $n \geq 2$, as was shown in [Fässler 2007; Magnani 2010]. 
More generally, based on recent results in [Wenger and Young 2010] the Lipschitz extension property holds for maps from any set with Assouad-Nagata dimension less than or equal to $n$ to any jet space group on $\mathbb{R}^{n}$. Notably, this implies the Lipschitz extension property for maps from $H_{k}$ to $H_{2 k+1}$.

2D. General Carnot groups. We now explain how the constructions performed in Section 2C on the Heisenberg group can be generalized to work on other Carnot groups. We need a notion of discretization (already used implicitly in the decomposition in Section 2C1).

Definition 2.11. Let $G$ be a Carnot group whose Lie algebra $\mathfrak{g}$ is graded as

$$
\mathfrak{g}=\bigoplus_{j=1}^{d} \mathfrak{g}_{j}
$$

Write $m_{j}$ as the vector space dimension of $\mathfrak{g}_{j}$ for $1 \leq j \leq d$. We say that $G$ is discretizable if for $1 \leq j \leq d$ there exist collections

$$
\left\{X_{(j, i)}\right\}_{i=1}^{m_{j}} \in \mathfrak{g}_{j} \quad \text { and } \quad\left\{g_{(j, i)}\right\}_{i=1}^{m_{j}} \in G, \quad \text { with } \exp \left(X_{(j, i)}\right)=g_{(j, i)},
$$

and subgroups

$$
H_{j} \leq G, \quad \text { where } H_{j}=\left\langle\left\{g_{\left(j^{\prime}, i\right)}\right\}_{1 \leq i \leq m_{j^{\prime}}, j \leq j^{\prime} \leq d}\right\rangle,
$$

such that $\left\{X_{(j, i)}\right\}_{i=1}^{m_{j}}$ spans $\mathfrak{g}_{j}$ as a vector space and, writing $G^{\prime}=\left\langle\left\{g_{(1, i)}\right\}_{i=1}^{m_{1}}\right\rangle$ and $G_{j}=\left\langle\left\{\exp \left(\mathfrak{g}_{j^{\prime}}\right)\right\}_{j^{\prime} \geq j}\right\rangle$,

$$
G^{\prime} \text { is a discrete subgroup with } G^{\prime} \cap G_{j}=H_{j} .
$$

In this setting, we say that $G^{\prime}$ is the discretization of $G$.

Examples of discretizable Carnot groups include Heisenberg groups, Euclidean spaces, and jet spaces. For example, we can take the discrete Heisenberg groups as the discretization of the Heisenberg groups.

If $G$ is discretizable, the method in [Christ 1990] can be followed as in Section 2C1 to create a dyadic decomposition, with $B_{0}$ now defined to be the discretization $G^{\prime}$. Although the scaling constant used to create $B_{n}$ from $B_{0}$ (which was $10^{-n}$ in the case of Heisenberg groups) depends on the specific Carnot group itself (in particular, it depends on the relationship between the coordinates of an arbitrary point $g$ and $d_{C C}(g, 0)$; compare Theorem 2.10 in [Montgomery 2002]), the procedure for Heisenberg groups can otherwise be copied exactly to create a dyadic decomposition for $G$ into dyadic "cubes". Because the base cube for our construction will still be a cube based at the origin of scale zero, we can still refer to it as $Q(0,0)$. With our new dyadic decomposition in hand, we can also copy the construction of the $C_{\beta}$ and $C_{\beta}^{\prime}$ in Section $2 \mathrm{C} 2$ in the setting of our discretizable group $G$. 
Finally, we observe that the results in Sections 2C3 and 2C4 (which involved differentiability and weak convergence) used no properties specific to Heisenberg groups. Therefore, the results in Sections 2C3 and 2C4 carry over just as well to maps from one Carnot group to another. Actually, Fact 2.8 in Section 2C3 was already stated and proved in terms of Carnot groups.

2E. Proof of main theorem. In what follows, $H^{k}$ and $h^{k}$ shall refer to Hausdorff $k$-dimensional measure and Hausdorff $k$-dimensional content, respectively (both of which we define with respect to Carnot-Carathéodory distance).

Our goal is to prove the following theorem.

Theorem 2.12. Let $G$ be a discretizable Carnot group of homogeneous dimension $k$ and let $H$ be another Carnot group. Suppose $F: Q(0,0) \subset G \rightarrow H$ is Lipschitz. If $\delta>0$, there exists a positive integer $N$ and subsets $Z, X_{1}, \ldots, X_{N}$ of $Q(0,0)$ such that

$$
\begin{gathered}
h^{k}(F(Z))<\delta, \\
Z \cup X_{1} \cup \cdots \cup X_{N}=Q(0,0),
\end{gathered}
$$

and $F$ is bilipschitz on each $X_{i}$. Furthermore, $N$ and the bilipschitz coefficients of the $F \mid X_{i}$ depend only on the groups $G$ and $H, \delta$, and the Lipschitz coefficient of $F$, and not on the map $F$ itself.

Before beginning the proof, we shall introduce two notions of nearness.

Definition 2.13. Suppose $Q(x, \alpha)$ and $Q(y, \alpha)$ are elements of the decomposition from $2 \mathrm{C} 1$ of a discretizable Carnot group into cubes of the same scale. We say that $Q(x, \alpha)$ and $Q(y, \alpha)$ are adjacent if the distance from $Q(x, \alpha)$ to $Q(y, \alpha)$ is bounded above by the diameter of $Q(x, \alpha)$.

Note that two coincident cubes of the same scale are considered adjacent.

Definition 2.14. Suppose $Q(x, \alpha)$ and $Q(y, \alpha)$ are elements of the decomposition of a discretizable Carnot group into cubes of the same scale. We say that $Q(x, \alpha)$ and $Q(y, \alpha)$ are semiadjacent if $Q(x, \alpha)$ and $Q(y, \alpha)$ are not adjacent and the parents of $Q(x, \alpha)$ and $Q(y, \alpha)$ are not adjacent, but the grandparents of $Q(x, \alpha)$ and $Q(y, \alpha)$ are adjacent.

Turning to the proof of Theorem 2.12, we begin by establishing some further notation and normalizations.

Let $E$ be the ratio of the diameter of an arbitrary "cube" to the diameter of one of its "children" using Carnot-Carathéodory distance. For example, if $G$ is a Heisenberg group (using exactly the "cube" decomposition from Section 2C1), then $E=10$. 
Using the Carnot-Carathéodory distance, we set

$$
\theta=\operatorname{diam}(Q(0,0)) \text {. }
$$

Also, we let $0<L_{1}<L_{2}<\infty$ be constants such that if $Q$ and $Q^{\prime}$ are semiadjacent,

$$
L_{1} \operatorname{diam}(Q)<d\left(Q, Q^{\prime}\right)<L_{2} \operatorname{diam}(Q) .
$$

We note that $L_{1}$ and $L_{2}$ only depend on $G$, not $Q$ or $Q^{\prime}$.

In addition, we may assume that $F$ is 1-Lipschitz and that there exists $\eta>0$ such that $F$ is defined on the dilation $\delta_{1+\eta} Q(0,0)$. For convenience we scale Hausdorff measure so that $|Q(0,0)|=1$ where $|S|$ denotes the Hausdorff measure of $S$.

Finally, we let $W$ be a positive integer such that every cube $Q^{\prime}$ of scale $W-10$ such that $Q^{\prime} \cap Q(0,0) \neq \varnothing$ satisfies $Q^{\prime} \subset \delta_{1+\eta} Q(0,0)$. Throughout this proof, we will be focusing primarily on subcubes of $Q(0,0)$ of scale at least $W$.

With our notation and normalizations set up, we prove the following proposition, which provides a partial wavelet decomposition of the linear map $M F$ induced by the Pansu differential $D F$ of $F$.

Proposition 2.15. Suppose $1 \geq \epsilon>0$. There exists $n, C>0$ such that if $\alpha \geq W$ and $Q:=Q(a, \alpha)$ and $Q^{\prime}:=Q(b, \alpha)$ are semiadjacent cubes with

$$
h^{k}(F(Q))>\epsilon E^{-k \alpha}
$$

and

$$
h^{k}\left(F\left(Q^{\prime}\right)\right)>\epsilon E^{-k \alpha}
$$

but

$$
d_{C C}\left(F(Q), F\left(Q^{\prime}\right)\right) \leq \frac{1}{2} \epsilon L_{1} \theta E^{-\alpha}
$$

then there exists $\beta \in[\alpha-4, \alpha+n]$ and $f_{Q, Q^{\prime}} \in C_{\beta}^{\prime}$ and integers $i, j$ such that

$$
\frac{\left|\left\langle(M F)_{i, j}, f_{Q, Q^{\prime}}\right\rangle\right|}{\left|\left\langle f_{Q, Q^{\prime}}, f_{Q, Q^{\prime}}\right\rangle\right|} \geq C|Q|^{1 / 2},
$$

where $C_{\beta}^{\prime}$ is the space defined in Section $2 C 2$ and MF is the matrix of horizontal components of the Pansu differential DF.

Further, $C$ only depends on $G, H$, and $\epsilon$ (and, in particular, not on the specific choice of $F$ ).

Also, the inner product in (4) is taken with respect to $L^{2}(G)$; it equals

$$
\int_{G}(M F)_{i, j} f_{Q, Q^{\prime}} d \mu
$$

where $\mu$ is Haar measure on $G$ scaled so that $\mu(Q(0,0))=1$. 
We also note that the number of possible candidates for $f_{Q, Q^{\prime}}$ for a given $Q$ is uniformly bounded, with a bound that depends only on the specific groups $G$ and $H$. Proof. Assume the contrary. Then, for each $n$ there exists a 1-Lipschitz map $F_{n}$ and semiadjacent cubes $Q\left(a_{n}, \alpha_{n}\right)$ and $Q\left(b_{n}, \alpha_{n}\right)$ such that

- $h^{k}\left(F_{n}\left(Q\left(a_{n}, \alpha_{n}\right)\right)\right)>\epsilon E^{-k \alpha_{n}}$,

- $h^{k}\left(F_{n}\left(Q\left(b_{n}, \alpha_{n}\right)\right)\right)>\epsilon E^{-k \alpha_{n}}$,

- $d_{C C}\left(F_{n}\left(Q\left(a_{n}, \alpha_{n}\right)\right), F_{n}\left(Q\left(b_{n}, \alpha_{n}\right)\right)\right)<\frac{1}{2} \epsilon L_{1} \theta E^{-\alpha_{n}}$, and

- $\int_{Q(0,0)} \psi f \leq 2^{-n}\left|Q\left(a_{n}, \alpha_{n}\right)\right|^{1 / 2}\|f\|_{L^{2}(Q(0,0))}^{2}$ whenever $\psi$ is a matrix entry of $M F_{n}$ and $f \in C_{\beta}^{\prime}$, where $\beta \in\left[\alpha_{n}-4, \alpha_{n}+n\right]$.

By rescaling and translating we may suppose

$$
Q\left(a_{n}, \alpha_{n}\right)=Q(a, \alpha)
$$

for all $n$ and by passing to a subsequence we suppose

$$
Q\left(b_{n}, \alpha_{n}\right)=Q(b, \alpha)
$$

for all $n$. Further, the Arzelà-Ascoli theorem lets us pass to another subsequence such that $F_{n}$ converges uniformly on $Q(0,0)$ to some Lipschitz map $F$. Moreover, by translation (we can do this because of the expanded $C^{\prime}$ families) we can suppose $Q(a, \alpha)$ and $Q(b, \alpha)$ have the same great-great-grandparent $Q(z, \alpha-4)$. By weak-star convergence, the restriction of each component of $M F$ to $Q(z, \alpha-4)$ is orthogonal to $C_{\beta}$ for $\beta>\alpha-4$ which implies that $M F$ is constant almost everywhere on $Q(z, \alpha-4)$. From this, the discussion in Section $2 \mathrm{C} 3$ lets us conclude that there exists a Lie group homomorphism $\phi$ such that $D F=\phi$ on $Q(z, \alpha-4)$ and further, there exist elements $g_{0} \in G, h_{0} \in H$ such that

$$
F(g)=h_{0} \phi\left(g_{0}^{-1} g\right)
$$

for all $g \in Q(z, \alpha-4)$. Further,

$$
h^{k}\left(F(Q(a, \alpha)) \geq \liminf _{n} h^{k}\left(F_{n}(Q(a, \alpha))\right) \geq \epsilon E^{-k \alpha}\right.
$$

because $F_{n}(Q(a, \alpha))$ is eventually contained in an arbitrarily small neighborhood of the closure $\overline{F(Q(a, \alpha))}$; such a neighborhood can have Hausdorff content arbitrarily close to $h^{k}(F(Q(a, \alpha)))$.

Working towards a contradiction, we next define the sequences of points $\left\{X_{n}\right\}$ and $\left\{Y_{n}\right\}$ such that

$$
X_{n} \in Q(a, \alpha), Y_{n} \in Q(b, \alpha)
$$

and

$$
d_{C C}\left(F_{n}\left(X_{n}\right), F_{n}\left(Y_{n}\right)\right) \leq \frac{1}{2} \epsilon L_{1} \theta E^{-\alpha}
$$


By the definition of sequential compactness, there exist points $a^{\prime} \in Q(a, \alpha), b^{\prime} \in$ $Q(b, \alpha)$ such that

$$
d_{C C}\left(F\left(a^{\prime}\right), F\left(b^{\prime}\right)\right) \leq \frac{1}{2} \epsilon L_{1} \theta E^{-\alpha} .
$$

However, because $Q(a, \alpha)$ and $Q(b, \alpha)$ are semiadjacent,

$$
d_{C C}\left(a^{\prime}, b^{\prime}\right) \geq L_{1} \theta E^{-\alpha} .
$$

Therefore, since (5) implies that the Pansu differential $D F$ of $F$ is defined everywhere and, in fact, is constant, the image of the Pansu differential $D F$ of $F$ in the direction of the tangent vector from $a^{\prime}$ to $b^{\prime}$ has magnitude at most $\frac{1}{2} \epsilon$. As $F$ is Lipschitz with coefficient 1 , this implies that

$$
h^{k}(F(Q(a, \alpha))) \leq|F(Q(a, \alpha))| \leq \frac{1}{2} \epsilon E^{-k \alpha} .
$$

The first inequality in (6) follows immediately from the fact that Hausdorff content is bounded above by Hausdorff measure. The second inequality is a direct consequence of the change-of-variables formula for Carnot groups (see the proof of Theorem 7 of [Vodopyanov and Ukhlov 1996], which can be directly adapted to this case).

As (6) contradicts our hypotheses, the proposition follows.

Armed with this proposition, our next goal is to show that a sufficiently large portion of our domain lies in finitely many such semiadjacent pairs.

Proposition 2.16. Let $\Omega$ be the set of all pairs of cubes which satisfy the hypotheses of Proposition 2.15 and let

$$
\phi(x)=\#\left\{\omega=\left(Q, Q^{\prime}\right) \in \Omega: x \in Q \cup Q^{\prime}\right\} .
$$

Suppose $N>0$; then there exists a constant $K^{\prime}$ depending only $G, H$, and $\epsilon$ such that

$$
|\{x: \phi(x) \geq N\}| \leq K^{\prime} N^{-1} .
$$

Proof. If $\left(Q, Q^{\prime}\right) \in \Omega$, Proposition 2.15 gives us a wavelet function $f_{Q, Q^{\prime}}$ corresponding to $\left(Q, Q^{\prime}\right)$ such that the projection of $M F$ onto $f_{Q, Q^{\prime}}$ had $L^{2}$ magnitude at least $C \epsilon|Q|^{1 / 2}$. However, only a bounded number of pairs of cubes can be assigned a given wavelet function in this way. This is because of the control that Proposition 2.15 gives to both the scale and support of $f_{Q, Q^{\prime}}$ in terms of the scale and location of $Q$. Now, we seek to show that

$$
1 \succeq \sum_{\left(Q, Q^{\prime}\right) \in \Omega}|Q|
$$

where the implied multiplicative constant only depends on $G, H$, and $\epsilon$.

Because $F$ is 1-Lipschitz, we can replace the constant 1 on the left hand side with $\|M F\|_{2}^{2}$. Next, for any specific pair $\left(Q, Q^{\prime}\right)$ in our sum, we let $\pi_{\left(Q, Q^{\prime}\right)}(M F)$ 
be the orthogonal projection of $M F$ onto $f_{Q, Q^{\prime}}$. By Proposition 2.15,

$$
\left\|\pi_{\left(Q, Q^{\prime}\right)}(M F)\right\|_{2} \geq C \epsilon|Q|^{1 / 2} ;
$$

in other words,

$$
\int\left|\pi_{\left(Q, Q^{\prime}\right)}(M F)\right|^{2} \geq C^{2} \epsilon^{2}|Q| .
$$

Summing this over $\Omega$ gives us indeed that

$$
1 \succeq\|M F\|_{2}^{2} \succeq \sum_{\left(Q, Q^{\prime}\right) \in \Omega}|Q|
$$

because the $f_{Q, Q^{\prime}}$ are approximately orthogonal and a given wavelet function can only appear in the sum a bounded number of times. However,

$$
\int \phi=\sum_{\left(Q, Q^{\prime}\right) \in \Omega}|Q|,
$$

so Chebyshev's inequality therefore tells us that

$$
S_{N}=\{x: \phi(x) \geq N\}
$$

has

$$
\left|S_{N}\right| \preceq N^{-1},
$$

which proves the proposition.

Proof of theorem. We complete the theorem through an infinite series of iterations as in [Jones 1988]. This process is divided into stages (indexed by $\alpha \geq 0$ ); at stage $\alpha$ we assign each point $x$ of each subcube of $Q(0,0)$ of scale $\alpha$ a label $x_{\alpha}$, i.e., a finite string of zeroes and ones, such that every point in a fixed cube of scale $\alpha$ has the same label.

At stage 0 we apply a leading digit of 0 to every point in the base cube. In other words, for each $x \in Q(0,0)$, we set $x_{0}=0$. Also, we define $Z_{0}=\varnothing$ for future reference.

For $0<\alpha$, we begin by defining the garbage set $Z_{\alpha}$ by letting $S_{\alpha}$ be the collection of all cubes $Q$ of scale $\alpha+W$ such that

$$
|F(Q)| \leq \delta E^{-k(\alpha+W)}
$$

and set $Z_{\alpha}=S_{\alpha} \cup Z_{\alpha-1}$.

Next, we run through each pair of cubes at scale $\alpha+W$ which lie in $Q(0,0) \backslash Z_{\alpha}$ and which satisfy the hypotheses of Proposition 2.16 with $\epsilon=\frac{1}{100} \delta$. Supposing that there are $n_{\alpha}$ such pairs $\left(Q_{1}, Q_{1}^{\prime}\right), \ldots,\left(Q_{n_{\alpha}}, Q_{n_{\alpha}}^{\prime}\right)$, we will inductively define the labels $x_{(\alpha, m)}$ for $m=0,1, \ldots, n_{\alpha}$ as follows:

First, $x_{(\alpha, 0)}=x_{\alpha-1}$ for each $x \in Q(0,0) \backslash Z_{\alpha}$. Then, for $m>0$ we define $x_{(\alpha, m)}=x_{(\alpha, m-1)}$ for $x \notin Q_{m} \cup Q_{m}^{\prime}$. We note that $x_{(\alpha, m-1)}$, when viewed as a 
function on $Q(0,0) \backslash Z_{\alpha}$, is constant at a value (call it $z_{1}$, and let $y_{1}$ be its length) on $Q_{m}$ and at a possibly different value (call it $z_{2}$, and let $y_{2}$ be its length) on $Q_{m}^{\prime}$; without loss of generality we may assume that $y_{1} \geq y_{2}$. There are several cases to consider:

(I) If $y_{1}=y_{2}$ and $z_{1} \neq z_{2}$ we simply define $x_{(\alpha, m)}=x_{(\alpha, m-1)}$ on both $Q_{m}$ and $Q_{m}^{\prime}$.

(II) If $y_{1}=y_{2}$ and $z_{1}=z_{2}$ we then let $x_{(\alpha, m)}$ be equal to the string created by adding a 0 to the end of $x_{(\alpha, m-1)}$ on $Q_{m}$ and the string created by adding a 1 to the end of $x_{(\alpha, m-1)}$ on $Q_{m}^{\prime}$.

(III) If $y_{1}>y_{2}$ and $z_{2}$ is not the first $y_{2}$ digits of $z_{1}$ we simply define $x_{(\alpha, m)}=x_{(\alpha, m-1)}$ on both $Q_{m}$ and $Q_{m}^{\prime}$.

(IV) If $y_{1}>y_{2}$ and $z_{2}$ is the first $y_{2}$ digits of $z_{1}$, we let define $x_{(\alpha, m)}=x_{(\alpha, m-1)}$ on $Q_{m}$; on $Q_{m}^{\prime}$ we let $y^{\prime}$ be the element of $\{0,1\}$ that is not the $\left(y_{2}+1\right)$-th digit of $z_{1}$ and define $x_{(\alpha, m)}$ on $Q_{m}^{\prime}$ to be the string created by adding $y^{\prime}$ to the end of $x_{(\alpha, m-1)}$.

Once we have finished this process for each cube, we define $x_{\alpha}=x_{\left(\alpha, n_{\alpha}\right)}$ on $Q(0,0) \backslash Z_{\alpha}$.

Now, defining $Y_{n}$ to be the set of all points $x$ such that $x_{\alpha}$ has length at least $n$ for some $\alpha$, we conclude from Proposition 2.16 that there exists $N$ such that

$$
\left|\left\{x \in Q(0,0) \backslash \bigcup_{\alpha} Z_{\alpha}: x \in Y_{N}\right\}\right|<\frac{1}{100} \delta
$$

we now define the set $Z=\bigcup_{\alpha} Z_{\alpha} \cup Y_{N}$.

If $x \in Q(0,0) \backslash Z$, the sequence $\left\{x_{\alpha}\right\}$ is eventually constant; denote its limiting value by $x_{\infty}$. Since there are at most $2^{n}$ strings of length $n$, there are at most $2^{N}$ possible values of $x_{\infty}$.

We finish by setting

$$
X_{w}=\left\{x \in Q(0,0) \backslash Z: x_{\infty}=w\right\}
$$

whenever $w$ is a string of zeroes and ones of length less than $N$. For each such $w, F \mid X_{w}$ must be bilipschitz (if not, there exist $x_{1}, x_{2} \in X_{w}$ and a pair of cubes $\left(Q, Q^{\prime}\right)$ satisfying the hypotheses of Proposition 2.15 such that $x_{1} \in Q, x_{2} \in Q^{\prime}$, contradicting the definition of $X_{w}$ ), proving the theorem.

\section{F. Consequences.}

Corollary 2.17. Suppose $A$ is an open subset of a discretizable Carnot group $G$ (with homogeneous dimension $k$ ), $H$ is another Carnot group, and $F: A \rightarrow H$ is Lipschitz, and $H^{k}(F(A))>0$. Then there exists a subset $B \subset A$ of positive $k$-dimensional Hausdorff measure such that $F$ restricted to $B$ is bilipschitz. 
Proof. We can express $A$ as a countable union of translates and dilates of the base cube $Q(0,0)$; by countable additivity of Hausdorff measure one of these cubes, which we call $C$, is sent by $F$ to a set $F(C)$ with $H^{k}(F(C))>0$. By rescaling we can suppose $C$ is the base cube $Q(0,0)$. The previous theorem divides this cube into the union of a "garbage" set $Z$ (consisting of those cubes whose image has measure too small, as well as those cubes which are in too many bad pairs), where $F(Z)$ can be taken to be arbitrarily small (say, with $h^{k}(F(Z))<\frac{1}{2} h^{k}(F(A))$ ) and a finite union of sets $F_{j}$ such that $F \mid F_{j}$ is bilipschitz for each $j$. Since $H^{k}\left(F\left(\bigcup_{j} F_{j}\right)\right)>0$, there exists some $j$ where $\left|F_{j}\right|>0$ and we let $B=F_{j}$.

If one assumed that $H^{k}(A)<\infty$, looking closely at the shape of $A$ would allow us to conclude above that the measure of $B$ and the bilipschitz constant of $F$ would depend only on $G, H, A$, the Lipschitz coefficient of $F$, and the $k$ dimensional Hausdorff content of $F(A)$.

Restricting attention to the first Heisenberg group $H_{1}$, we use this corollary to show that if we only consider maps whose domains are open, two questions from [Heinonen and Semmes 1997] are equivalent. To begin we need two more definitions.

Definition 2.18. Suppose $Q_{1}$ and $Q_{2}$ are metric spaces with Hausdorff dimension $k$. We say that $Q_{1}$ looks down on $Q_{2}$ if there exists a Lipschitz function $f$ from some subset of $Q_{1}$ to $Q_{2}$ such that the image of $f$ has nonzero Hausdorff $k$-measure.

Definition 2.19. Suppose $Q$ is a metric space with Hausdorff dimension $k$. We say that $Q$ is minimal in looking down if whenever $Q^{\prime}$ is a metric space with Hausdorff dimension $k$ such that $Q$ looks down on $Q^{\prime}, Q^{\prime}$ also looks down on $Q$.

(Note that this definition is formulated differently from the one in [Heinonen and Semmes 1997].)

Question 22 in [Heinonen and Semmes 1997] asks whether the first Heisenberg group is minimal in looking down and Question 24 asks if every Lipschitz map from $H_{1}$ to a metric space with nontrivial Hausdorff 4-measure is bilipschitz on some subset with positive Hausdorff 4-measure.

Clearly 24 implies 22 . However, we now know from the corollary that 22 implies 24 when only looking at maps from open sets. This is true because (assuming $H_{1}$ is minimal in looking down) if $F: E \subset H_{1} \rightarrow X$ is Lipschitz and $H^{4}(F(E))>0$ then, letting $G: X \rightarrow H_{1}$ be another Lipschitz map with $H^{4}(G(X))>0$ (and supposing, by restricting images, that $X=F(E)), G \circ F$ satisfies the conditions of the corollary and therefore is bilipschitz on some subset $E^{\prime} \subset E$ with $\left|E^{\prime}\right|>0$. On this set, we therefore have that $F$ is invertible with inverse $(G \circ F)^{-1} \circ G$, which is clearly Lipschitz, which therefore implies that $F \mid E^{\prime}$ is bilipschitz. Because $F$ was arbitrary, we can conclude that Question 24, when restricted to maps defined on open sets, is equivalent to Question 22. 
Raanan Schul recently proved a statement corresponding to Question 24 for maps where the domain is Euclidean in [Schul 2009]. In particular, he showed that if $F$ is a Lipschitz function from the $k$-dimensional unit cube $[0,1]^{k}$ into a general metric space, one has the decomposition

$$
[0,1]^{k}=G \cup \bigcup_{j=1}^{n} F_{j},
$$

where $F(G)$ has arbitrarily small $k$-dimensional Hausdorff content and $F$ is bilipschitz on each of the $F_{j}$. The main reason why Schul's argument does not generalize to this setting is the dearth of rectifiable curves passing through a given point in a general Carnot group. For example, although the first Heisenberg group has Hausdorff dimension 4, the space of horizontal tangents to rectifiable curves through a given point in that group has dimension two.

We finish this section by discussing the question of Jones-style decompositions for Lipschitz maps on Carnot groups. Just as in [Jones 1988], my argument for the main theorem actually implies the following stronger statement:

Corollary 2.20. Suppose $U$ is a bounded open subset of a discretizable Carnot group $G$ with Hausdorff dimension $Q, H$ is another Carnot group, $F: U \rightarrow H$ is Lipschitz, and $\epsilon>0$. Then there exists a finite collection $\left\{A_{i}\right\}$ of subsets of $U$ such that each restriction $F \mid A_{i}$ is bilipschitz and

$$
h^{Q}\left(F\left(U \backslash \bigcup_{i} A_{i}\right)\right)<\epsilon .
$$

For unbounded open subsets of discretizable Carnot groups a diagonalization argument yields the following.

Corollary 2.21. Suppose $U$ is an open subset of a discretizable Carnot group $G$ with Hausdorff dimension $Q, H$ is another Carnot group and $F: U \rightarrow H$ is Lipschitz. Then there exists a countable collection $\left\{A_{i}\right\}$ of subsets of $U$ such that each restriction $F \mid A_{i}$ is bilipschitz and

$$
h^{Q}\left(F\left(U \backslash \bigcup_{i} A_{i}\right)\right)=0 .
$$

A natural generalization of the above results is in the setting of subriemannian manifolds, defined below.

Definition 2.22. A subriemannian manifold is a triple $(M, \Delta, g)$ where $M$ is a smooth manifold, $\Delta$ is a distribution (i.e., subbundle of the tangent bundle $T M$ ) on $M$ which is smooth and satisfies the property that for each $p \in M,(T M)_{p}$ is generated as a Lie algebra by $\Delta_{p}$, and $g$ is a smooth section of positive-definite quadratic forms on $\Delta$ (i.e., $g_{p}$ defines an inner product on $\Delta_{p}$ which varies smoothly in $p$ ). 
Recall [Varadarajan 1984] that the set $S$ is said to generate a Lie algebra $\mathfrak{g}$ if the set of finite Lie brackets of elements of $S$ spans $\mathfrak{g}$ as a vector space.

We shall consider $M$ to be naturally equipped with a metric $d_{C C}$ defined as follows: for $x, y \in M$,

$$
d_{C C}(x, y)=\inf _{\gamma \in \Gamma_{x, y}} \int_{0}^{1} \sqrt{g\left(\gamma^{\prime}(t), \gamma^{\prime}(t)\right)} d t
$$

where $\Gamma_{x, y}$ is the family of all curves

$$
\gamma:[0,1] \rightarrow M
$$

with $\gamma(0)=x, \gamma(1)=y$, and $\gamma^{\prime}(t) \in \Delta_{\gamma(t)}$ for all $t$.

Now, suppose $M$ and $N$ are subriemannian manifolds such that $M$ is locally bilipschitz equivalent to a discretizable Carnot group $G$ and $N$ is locally bilipschitz equivalent to a Carnot group $H$. Then Corollary 2.21 still holds if $G$ is replaced by $M$ and $H$ is replaced by $N$.

For example, $M$ and $N$ could both be ordinary riemannian manifolds. Because riemannian manifolds are locally bilipschitz equivalent to Euclidean spaces, where we have all five properties from Section 2, we can consider arbitrary subsets of $M$ instead of just open subsets. Thus we have the following corollary: if $M$ is a riemannian manifold, $A \subset M$ has Hausdorff dimension $k, N$ is another riemannian manifold, and $F: A \rightarrow N$ is Lipschitz with $H^{k}(F(A))>0$, then there exists a subset $B \subset A$ with $H^{k}(B)>0$ such that $f \mid B$ is bilipschitz.

Not all subriemannian manifolds are locally bilipschitz equivalent to Carnot groups, and hence we cannot replace $G$ and $H$ by arbitrary subriemannian manifolds in Corollary 2.21. In particular, we will show in Section 4B that Corollary 2.21 becomes false if $G$ and $H$ are replaced by the Grushin plane and the Euclidean plane, respectively.

\section{Hausdorff dimension of Lipschitz images}

We begin by observing the following corollary of the results in Section 2.

Corollary 3.1. Assume A is an open subset of a discretizable Carnot group $G$ with homogeneous dimension $k$, assume $H$ is another Carnot group, and let $f: A \rightarrow H$ be a Lipschitz map such that $H^{k}(f(A))>0$. Then there exists an injective Lie group homomorphism from $G$ to $H$.

Proof. By the preceding results, $f$ is bilipschitz on some $B \subset A$ with positive $k$-dimensional Hausdorff measure. Then the Pansu differential of $f$ at any Lebesgue point of $B$ gives the desired homomorphism. 
Because the converse of this result is trivial (the Lie group homomorphism in question is locally Lipschitz), Corollary 3.1 reduces the question of whether one Carnot group "looks down" on another to a question about the groups' Lie algebras.

An easy consequence of Corollary 3.1 is that if $G$ is a discretizable nonabelian Carnot group with homogeneous dimension $k$ and $U \subset G$ then every Lipschitz image of $U$ in any Euclidean space has zero $k$-dimensional Hausdorff measure. This follows because there are no injective group homomorphisms from a nonabelian group to an abelian group. In fact, for this consequence we need not assume $U$ is open here because the image space, Euclidean space, has the Lipschitz extension property.

Despite having Hausdorff measure $k$-measure zero, the Lipschitz image of $U$ in $\mathbb{R}^{k}$ can still be quite large. For example, we have the following theorem, which answers a question asked by Le Donne (personal communication, 2009):

Theorem 3.2. Suppose that $G$ is a discretizable Carnot group with homogeneous dimension $k$, and let $\epsilon>0$. There exists a bounded open $U \subset G$ and a Lipschitz map $F: U \rightarrow \mathbb{R}^{k}$ such that $H^{k-\epsilon}(F(U))>0$.

Proof. As in our results in Section 2, we illustrate the case $G=H^{1}$ in detail and remark that the construction is analogous for the general case. The construction is based on the procedure from [Kaufman 1979].

We begin by setting

$$
\gamma=16^{1 /(\epsilon-4)},
$$

which tells us that $\gamma<\frac{1}{2}$ and $\log _{\gamma^{-1}} 16=4-\epsilon$. We next fix $\beta \in\left[\gamma, \frac{1}{2}\right)$ and define

$$
\lambda=\frac{20}{\frac{1}{4}-\beta^{2}} ;
$$

in particular,

$$
\lambda\left(\frac{1}{4}-\beta^{2}\right)=20>10 .
$$

With this data, we then set our initial box

$$
I^{0}=[-1,1] \times[-1,1] \times[-\lambda, \lambda] \subset H^{1}
$$

and define $I^{1}$ to be the union of the sixteen boxes

$$
(a, b, c) \cdot \delta_{\beta} I^{0}
$$

where

$$
a \in\left\{-\frac{1}{2}, \frac{1}{2}\right\}, \quad b \in\left\{-\frac{1}{2}, \frac{1}{2}\right\}, \quad c \in\left\{-\frac{3}{4} \lambda,-\frac{1}{4} \lambda, \frac{1}{4} \lambda, \frac{3}{4} \lambda\right\} .
$$

We arbitrarily label these boxes $I_{j}^{1}$ for $j=1, \ldots, 16$. 
The point of this construction is to find $\eta>0$ such that

$$
d_{C C}\left(I_{j}^{1}, I_{k}^{1}\right)>\eta \quad \text { for } j \neq k
$$

and

$$
d_{C C}\left(I_{j}^{1}, \delta\left(I^{0}\right)\right)>\eta \quad \text { for all } j .
$$

Clearly, if two of the boxes in $I^{1}$ have different horizontal components, then they are at least $1-2 \beta$ apart; similarly, every box in $I^{1}$ is at a distance of exactly $\frac{1}{2}-\beta$ away from the nearest horizontal edge of $I^{0}$.

The only issue is vertical distance. To find the minimum distance between a vertical edge of $I^{0}$ and a box in $I^{1}$, it suffices to consider a box in $I^{1}$ where $c=-\frac{3}{4} \lambda$ and look at the bottom edge of $I^{0}$. Every point on the bottom edge of such a box has a vertical coordinate which is at least

$$
-\frac{3}{4} \lambda-\beta^{2} \lambda-2 \cdot \frac{1}{2} \beta>-\lambda+10-2=-\lambda+8 .
$$

Now, we recall that if $g=\left(x_{1}, y_{1}, 0\right)$ and $h=\left(x_{2}, y_{2}, 0\right)$ are points in $H^{1}$ with $x_{1}, y_{1}, x_{2}, y_{2} \in[-1,1]$, then writing the product $g^{-1} h$ as $\left(x_{3}, y_{3}, z_{3}\right)$ we note that $\left|z_{3}\right|<2$.

Consequently, if $p=\left(p_{1}, p_{2}, p_{3}\right)$ is a point in $I^{1}$ and $q=\left(q_{1}, q_{2},-\lambda\right)$ is a point on the bottom edge of $I^{0}$, we note that the vertical coordinate of $p^{-1} q$ is at most

$$
-(-\lambda+8)-\lambda+2=-6,
$$

which implies that vertical edges of $I^{0}$ will be separated from boxes in $I^{1}$ by at least 6 units.

Similarly, looking at two boxes in $I^{1}$ with the same horizontal component (e.g., let $A$ be such a box with $c=-\frac{3}{4} \lambda$ and $B$ be such a box with $c=-\frac{1}{4} \lambda$ ), the vertical coordinate of points in $A$ are at most $-\frac{1}{2} \lambda-8$ and the vertical coordinate of points in $B$ are at least $-\frac{1}{2} \lambda+8$. Therefore, whenever $a \in A$ and $b \in B$, the vertical coordinate of $a^{-1} b$ is at least

$$
\left(-\frac{1}{2} \lambda+8\right)-\left(-\frac{1}{2} \lambda-8\right)-2=14
$$

implying a separation of 14 between any two such boxes.

In subsequent stages we replace each box of the form

$$
p \cdot \delta_{\mu} I^{0}
$$

(there are $16^{k}$ such boxes in stage $k$; at this stage $\mu=\beta^{k}$ ) with the sixteen boxes

$$
p \cdot \delta_{\mu}(a, b, c) \cdot \delta_{\beta \mu}\left(I^{0}\right)
$$

and denote by $I^{k}$ the union of all the boxes produced in stage $k$. 
In stage $k$, each box has a label of the form $I_{\left(a_{1}, \ldots, a_{k}\right)}^{k}$ where each $a_{i}$ ranges from 1 to 16 ; we extend this process to stage $k+1$ by labeling the subboxes from $I_{\left(a_{1}, \ldots, a_{k}\right)}^{k}$ as $I_{\left(a_{1}, \ldots, a_{k}, v\right)}^{k+1}$ where $v=1,2, \ldots, 16$. The intersection of the $I^{k}$ 's, to be defined as $I$, is a Cantor set in $H^{1}$ of dimension

$$
\log _{\beta^{-1}} 16 \geq 4-\epsilon .
$$

Each point $x \in I$ has a unique label of the form $\left(a_{1}, \ldots, a_{n}, \ldots\right)$ (where each $a_{i}$ ranges from 1 to 16) such that for each $n \in \mathbb{N}, x \in I_{\left(a_{1}, \ldots, a_{n}\right)}^{n}$; if $v=\left(a_{1}, \ldots, a_{n}, \ldots\right)$ and $w=\left(b_{1}, \ldots, b_{n}, \ldots\right)$ with $m$ being the smallest integer where $a_{m} \neq b_{m}$, the distance between the points corresponding to $v$ and $w$ is (up to a multiplicative constant independent of $m$ ) equal to $\beta^{m}$.

Similarly, we set $J^{0}$ to be the box $[-1,1]^{4}$ in Euclidean space $\mathbb{R}^{4}$ and $J^{1}$ to be the union of the sixteen boxes

$$
(a, b, c, d)+\gamma I^{0}
$$

where $a, b, c, d$ can each equal $-\frac{1}{2}$ or $\frac{1}{2}$. We arbitrarily label these boxes $J_{j}^{1}$ for $j=1, \ldots, 16$.

The point of this construction is now to find $\eta^{\prime}>0$ such that

$$
d\left(J_{j}^{1}, J_{k}^{1}\right)>\eta^{\prime} \quad \text { for } j \neq k
$$

and

$$
d\left(J_{j}^{1}, \delta\left(J^{0}\right)\right)>\eta^{\prime} \quad \text { for all } j,
$$

where the distance above is Euclidean.

Clearly, any two of the boxes in $J^{1}$ are at least $1-2 \gamma$ apart; similarly, each such box is at a distance of exactly $\frac{1}{2}-\gamma$ away from the boundary of $J^{0}$.

In subsequent stages we replace the box

$$
p+v J^{0}
$$

with the sixteen boxes

$$
p+v\left((a, b, c, d)+\gamma J^{0}\right)
$$

and denote by $J^{k}$ the union of all boxes produced in stage $k$. Note that at stage $k$, $v=\gamma^{k}$.

In stage $k$, each box has a label of the form $J_{\left(a_{1}, \ldots, a_{k}\right)}^{k}$ where each $a_{i}$ ranges from 1 to 16 ; we extend this process to stage $k+1$ by labeling the subboxes from $J_{\left(a_{1}, \ldots, a_{k}\right)}^{k}$ as $J_{\left(a_{1}, \ldots, a_{k}, v\right)}^{k+1}$ where $v=1,2, \ldots, 16$. The intersection of the $J^{k}$ s, to be defined as $J$, is a Cantor set in $\mathbb{R}^{4}$ of dimension

$$
\log _{\gamma^{-1}} 16=4-\epsilon
$$


Each point $x \in J$ has a unique label of the form $\left(a_{1}, \ldots, a_{n}, \ldots\right)$ (where each $a_{i}$ ranges from 1 to 16) such that for each $n \in \mathbb{N}, x \in J_{\left(a_{1}, \ldots, a_{n}\right)}^{n}$; if $v=\left(a_{1}, \ldots, a_{n}, \ldots\right)$ and $w=\left(b_{1}, \ldots, b_{n}, \ldots\right)$ with $m$ being the smallest integer where $a_{m} \neq b_{m}$, the distance between the points corresponding to $v$ and $w$ is (up to a multiplicative constant independent of $m$ ) equal to $\gamma^{m}$.

We can define a Lipschitz map $F$ from $I^{0} \subset H^{1}$ to $\mathbb{R}^{4}$ whose image contains $J$ (and therefore has Hausdorff dimension $\log _{\gamma^{-1}}(16)$ ) via the following three-step process.

Step 1. Map $I$ to $J$. This is done by mapping a point in $I$ with a label of the form $\left.\overline{\left(a_{1}, \ldots\right.}, a_{n}, \ldots\right)$ to the point with the same label in $J$. By construction, one notes that if $\beta=\gamma$ then this map is bilipschitz.

Step 2. For each ordered $n$-tuple $\left(a_{1}, \ldots, a_{n}\right)$ with each $a_{i}$ in $\{1, \ldots, 16\}$ (this includes the zero-tuple, where we would be mapping the boundary of $I^{0}$ ) we choose a point $p_{\left(a_{1}, \ldots, a_{n}\right)}$ in $J_{\left(a_{1}, \ldots, a_{n}\right)}^{n}$ and then send all of the points in the boundary of $I_{\left(a_{1}, \ldots, a_{n}\right)}^{n}$ to $p_{\left(a_{1}, \ldots, a_{n}\right)}$.

Step 3. The remaining region of $I^{0}$ consists of sets of the form $S_{\left(a_{1}, \ldots, a_{n}\right)}^{n}$ defined as the set of all points in $I_{\left(a_{1}, \ldots, a_{n}\right)}^{n}$ which do not lie in $I_{\left(a_{1}, \ldots, a_{n}, v\right)}^{n+1}$ for $v=1,2, \ldots, 16$. The closure of this region includes the boundary of $I_{\left(a_{1}, \ldots, a_{n}\right)}^{n}$ and of $I_{\left(a_{1}, \ldots, a_{n}, v\right)}^{n+1}$ for $v=1, \ldots, 16$. Fixing $\left(a_{1}, \ldots, a_{n}\right)$ (we may work on each $S_{\left(a_{1}, \ldots, a_{n}\right)}^{n}$ separately) we define the map $f$ from the interval $[0,16]$ to $\mathbb{R}^{4}$ to be a smooth function sending 0 to $p_{\left(a_{1}, \ldots, a_{n}\right)}$ and $v=1, \ldots, 16$ to $p_{\left(a_{1}, \ldots, a_{n}, v\right)}$. We can suppose $f$ has Lipschitz norm comparable to $\gamma^{n}$. We then define $g$ to be a smooth, real-valued, Lipschitz function (with Lipschitz coefficient comparable to $\beta^{-n}$ ) on the closure of $S_{\left(a_{1}, \ldots, a_{n}\right)}^{n}$ which sends the boundary of $I_{\left(a_{1}, \ldots, a_{n}\right)}^{n}$ to 0 and the boundary of $I_{\left(a_{1}, \ldots, a_{n}, v\right)}^{n+1}$ to $v$. We can create such a $g$ by the Whitney extension theorem (the construction is more straightforward if we do not require smoothness). On the closure of $S_{\left(a_{1}, \ldots, a_{n}\right)}^{n}$ (the construction merely repeats the existing one on the boundary) set $F=f \circ g$; then $F \mid \overline{S_{\left(a_{1}, \ldots, a_{n}\right)}^{n}}$ has Lipschitz norm comparable to $\left(\frac{\gamma}{\beta}\right)^{n}$.

Note that if $\gamma<\beta,\left(\frac{\gamma}{\beta}\right)^{n}$ goes to zero as $n$ goes to infinity, which means that $F$ is differentiable (in the Pansu sense) at each point of $I$ with derivative zero. Further, by construction $F$ is $C^{1}$ outside of $I$ where the Pansu differential always has rank zero or one (and this differential approaches zero as we approach points of $I$ ); in fact, it is locally constant near the boundaries of the relevant cubes if we use the Whitney extension, so the construction here is indeed an appropriate analogue of [Kaufman 1979].

In fact, because the constructed map is constant on the boundary of $I^{0}$, nesting appropriately rescaled examples of this form inside each other yield the following corollary. 
Corollary 3.3. Suppose that $G$ is a discretizable Carnot group with homogeneous dimension $k$. There exists a bounded open $U \subset G$ and a Lipschitz map $F: U \rightarrow \mathbb{R}^{k}$ such that $F(U)$ has Hausdorff dimension $k$.

\section{Counterexamples}

In this section we develop two counterexamples to show why Carnot group structure, or something close to it, is necessary for the results of the previous two sections.

\section{A. A space-filling curve.}

Theorem 4.1. There exists an Ahlfors 2-regular metric space $X$ and a Lipschitz map $F: X \rightarrow \mathbb{R}^{2}$ such that $F(X)$ has positive 2-dimensional Hausdorff measure but $F$ is not bilipschitz on any set of positive 2-dimensional measure.

Proof. The function in question will be the space-filling curve $F$ from $[0,1]$ (equipped with the square root distance metric) to the unit square in $\mathbb{R}^{2}$ mentioned in Section 7.3 of [Stein and Shakarchi 2005]. Although this function is a surjective map of spaces with Hausdorff dimension 2 and Lipschitz, it is not bilipschitz on any subset with positive Hausdorff 2-measure. To see this, suppose that the space-filling curve $F$ is bilipschitz on a set $A$ with $H^{2}(A)>0$. As $F(A)$ has positive Lebesgue measure, it contains a point $x$ of Lebesgue density one. Letting $\epsilon>0$ there exists $\delta>0$ such that

$$
|B(x ; \delta) \cap F(A)|>(1-\epsilon)|B(x ; \delta)| .
$$

Writing out the binary expansion of the components of $x$ and of $\delta, B(x ; \delta)$ contains a dyadic cube $Q$ of side at least $\frac{1}{10} \delta$; since $\epsilon|B(x ; \delta)| \leq 1000 \epsilon|Q|$, we have

$$
|Q \cap F(A)|>(1-1000 \epsilon)|Q| .
$$

As $F$ is measure-preserving, letting $J$ be the preimage of $Q$ we conclude

$$
|J \cap A|>(1-1000 \epsilon)|J| .
$$

By rescaling and translating we can suppose $F$ is therefore bilipschitz on a set $A$ of Hausdorff 2-measure arbitrarily close to 1 (although the rescaled $F$ is not identical to our space-filling curve, it preserves all the relevant properties, such as being Lipschitz in the appropriate metric, measure-preserving, and sending a pair of points whose "square root" distance is at least $\frac{1}{2}$ to the same point).

Let $x, x^{\prime}$ be two points which are at least $\frac{1}{4}$ apart in Euclidean distance (and therefore $\frac{1}{2}$ away with respect to square root distance) such that $F(x)=F\left(x^{\prime}\right)$. We can suppose that $y, y^{\prime} \in A$ are arbitrarily close to $x, x^{\prime}$ respectively; therefore, $\left|y-y^{\prime}\right| \geq \frac{1}{4}$; however,

$$
\left|F(y)-F\left(y^{\prime}\right)\right| \leq|F(x)-F(y)|+\left|F\left(x^{\prime}\right)-F\left(y^{\prime}\right)\right|
$$


which can be made arbitrarily small by the Lipschitz property (all distances use the square root metric in the domain and the Euclidean metric in the image) showing that $F$ cannot be bilipschitz on $A$ with any coefficient.

In this example, the third and fourth properties (involving differentiability) from Section $2 \mathrm{C}$ fail. This suggests that some notion of differentiability is necessary for the results in [Jones 1988] to extend to other spaces.

\section{B. The Grushin plane.}

Theorem 4.2. There exists a 2-dimensional subriemannian manifold $M$ with Hausdorff dimension 2, an open $U \subset M$, and a Lipschitz map

$$
F: U \rightarrow \mathbb{R}^{2}
$$

which is not decomposable in the following sense: There does not exist a countable collection $\left\{A_{i}\right\}$ of sets such that

$$
H^{2}\left(F\left(U \backslash \bigcup_{i} A_{i}\right)\right)=0
$$

and $F \mid A_{i}$ is bilipschitz for each $i$.

Proof. We use the Grushin plane $M$ as our subriemannian manifold.

To construct the Grushin plane we define a riemannian metric on the following region of $\mathbb{R}^{2}:\{(x, y): y \neq 0\}$.

This metric is defined as $d s^{2}=d x^{2}+x^{-2} d y^{2}$. We then use this metric to induce a geodesic structure on all of $\mathbb{R}^{2}$, where a rectifiable curve must have horizontal tangent at each point that it crosses the $y$-axis.

One can observe that off of the vertical axis, the Grushin plane is locally bilipschitz to Euclidean space (but with a constant that blows up as we get closer to the axis). However, the distance between two points on the vertical axis is proportional to the square root of their Euclidean distance.

In other words, the Grushin plane is a union of a (disconnected) riemannian manifold and a line of Hausdorff dimension two, making it a subriemannian manifold of both Euclidean and Hausdorff dimension two.

To construct our counterexample, we consider an open neighborhood of the segment $S$ joining $(0,0)$ to $(0,1)$, say: $U_{\epsilon}=(-\epsilon, \epsilon) \times(-\epsilon, 1+\epsilon)$ for $\epsilon>0$. The space-filling curve previously constructed as in Chapter 7 of [Stein and Shakarchi 2005] has already been shown to be Lipschitz when defined as a function from a set which is bilipschitz to $S$ with image the unit square. We can extend this mapping to a Lipschitz mapping $F$ from $U_{\epsilon}$ to $\mathbb{R}^{2}$ by standard constructions (note the importance of having a Euclidean target space here).

However, there does not exist a countable collection of sets $A_{1}, \ldots, A_{n}, \ldots$ such that $G:=U_{\epsilon} \backslash \bigcup_{n} A_{n}$ is sent to a set of arbitrarily small Hausdorff content by $F$ and 
$F$ is bilipschitz when restricted to the $A_{n}$. This is because $A_{n} \cap S$ must be a nullset (by the previous arguments concerning the space-filling curve for each $G$ ) which implies that $G$ must contain almost all of $S$, in the sense of Hausdorff measure. Therefore, $F(G)$ must contain almost all of the unit square in the sense of Hausdorff measure (or Hausdorff content, which is equivalent in this case), producing our desired contradiction.

In this example, the first and second properties (involving homogeneity) from Section $2 \mathrm{C}$ fail, which suggests that some notion of homogeneity is also necessary for the results in [Jones 1988] to extend to other spaces.

\section{Acknowledgements}

The author would like to thank Raanan Schul for showing him Guy David's proof of the decomposition of Lipschitz functions from subsets of $\mathbb{R}^{m}$ to $\mathbb{R}^{n}$ into bilipschitz pieces. Further, the author would like to thank Jeremy Tyson for showing him the usefulness of Cantor set constructions for creating maps on Carnot groups.

\section{References}

[Balogh and Fässler 2009] Z. M. Balogh and K. S. Fässler, "Rectifiability and Lipschitz extensions into the Heisenberg group”, Math. Z. 263:3 (2009), 673-683. MR 2010j:53049 Zbl 1177.53032

[Brudnyi and Brudnyi 2007] A. Brudnyi and Y. Brudnyi, "Linear and nonlinear extensions of Lipschitz functions from subsets of metric spaces", Algebra i Analiz 19:3 (2007), 106-118. MR 2008m:26004 Zbl 1213.54040

[Capogna et al. 2007] L. Capogna, D. Danielli, S. D. Pauls, and J. T. Tyson, An introduction to the Heisenberg group and the sub-Riemannian isoperimetric problem, Progress in Mathematics 259, Birkhäuser, Basel, 2007. MR 2009a:53053 Zbl 1138.53003

[Christ 1990] M. Christ, "A $T(b)$ theorem with remarks on analytic capacity and the Cauchy integral", Colloq. Math. 60/61:2 (1990), 601-628. MR 92k:42020 Zbl 0758.42009

[David 1988] G. David, "Morceaux de graphes lipschitziens et intégrales singulières sur une surface", Rev. Mat. Iberoam. 4:1 (1988), 73-114. MR 90h:42026 Zbl 0696.42011

[David 1991] G. David, Wavelets and singular integrals on curves and surfaces, Lecture Notes in Mathematics 1465, Springer, Berlin, 1991. MR 92k:42021 Zbl 0764.42019

[David and Semmes 1993] G. David and S. Semmes, "Quantitative rectifiability and Lipschitz mappings”, Trans. Amer. Math. Soc. 337:2 (1993), 855-889. MR 93h:42015 Zbl 0792.49029

[Fässler 2007] K. Fässler, "Extending Lipschitz maps from Euclidean spaces into Heisenberg groups", Master's thesis, Universität Bern, 2007.

[Heinonen and Semmes 1997] J. Heinonen and S. Semmes, "Thirty-three yes or no questions about mappings, measures, and metrics", Conform. Geom. Dyn. 1 (1997), 1-12. MR 99h:28012 Zbl 0885.00006

[Jones 1988] P. W. Jones, "Lipschitz and bi-Lipschitz functions", Rev. Mat. Iberoam. 4:1 (1988), 115-121. MR 90h:26016 Zbl 0782.26007

[Kaufman 1979] R. Kaufman, "A singular map of a cube onto a square”, J. Differential Geom. 14:4 (1979), 593-594. MR 82a:26013 Zbl 0463.57011 
[Magnani 2010] V. Magnani, "Contact equations, Lipschitz extensions and isoperimetric inequalities", Calc. Var. Partial Differential Equations 39:1-2 (2010), 233-271. MR 2012g:49100 Zbl 1195.49053

[Montgomery 2002] R. Montgomery, A tour of subRiemannian geometries, their geodesics and applications, Mathematical Surveys and Monographs 91, Amer. Math. Soc., Providence, RI, 2002. MR 2002m:53045 Zbl 1044.53022

[Pansu 1989] P. Pansu, "Métriques de Carnot-Carathéodory et quasiisométries des espaces symétriques de rang un”, Ann. of Math. (2) 129:1 (1989), 1-60. MR 90e:53058 Zbl 0678.53042

[Rigot and Wenger 2010] S. Rigot and S. Wenger, "Lipschitz non-extension theorems into jet space Carnot groups”, Int. Math. Res. Not. 2010:18 (2010), 3633-3648. MR 2011f:53056 Zbl 1203.53028

[Schul 2009] R. Schul, "Bi-Lipschitz decomposition of Lipschitz functions into a metric space", Rev. Mat. Iberoam. 25:2 (2009), 521-531. MR 2011e:28007 Zbl 1228.28004

[Semmes 2000] S. Semmes, "Measure-preserving quality within mappings", Rev. Mat. Iberoam. 16:2 (2000), 363-458. MR 2001m:30029 Zbl 1041.42014

[Stein and Shakarchi 2005] E. M. Stein and R. Shakarchi, Real analysis: measure theory, integration, and Hilbert spaces, Princeton Lectures in Analysis 3, Princeton University Press, Princeton, NJ, 2005. MR 2005k:28024 Zbl 1081.28001

[Strichartz 1992] R. S. Strichartz, "Self-similarity on nilpotent Lie groups", pp. 123-157 in Geometric analysis (Philadelphia, PA, 1991), edited by E. L. Grinberg, Contemp. Math. 140, Amer. Math. Soc., Providence, RI, 1992. MR 94e:43011 Zbl 0797.43004

[Varadarajan 1984] V. S. Varadarajan, Lie groups, Lie algebras, and their representations, Graduate Texts in Mathematics 102, Springer, New York, 1984. MR 85e:22001 Zbl 0955.22500

[Vodopyanov and Ukhlov 1996] S. K. Vodopyanov and A. D.-O. Ukhlov, "Approximately differentiable transformations and change of variables on nilpotent groups", Sibirsk. Mat. Zh. 37:1 (1996), 70-89. In Russian; translated in Siberian Math. J. 37:1 (1996), 62-78. MR 97f:22011 Zbl 0870.43005

[Wenger and Young 2010] S. Wenger and R. Young, "Lipschitz extensions into jet space Carnot groups", Math. Res. Lett. 17:6 (2010), 1137-1149. MR 2011j:53048 Zbl 1222.53037

Received March 27, 2012. Revised August 14, 2012.

\section{WILLIAM MEYERSON}

Department of Mathematics and Statistics

HELSINGIN YLIOPISTO

FI-00014 HELSINKI

FINLAND

william.meyerson@helsinki.fi 


\title{
PACIFIC JOURNAL OF MATHEMATICS
}

\author{
msp.org/pjm
}

Founded in 1951 by E. F. Beckenbach (1906-1982) and F. Wolf (1904-1989)

\section{EDITORS}

V. S. Varadarajan (Managing Editor)

Department of Mathematics

University of California

Los Angeles, CA 90095-1555

pacific@math.ucla.edu

Paul Balmer

Department of Mathematics

University of California

Los Angeles, CA 90095-1555

balmer@math.ucla.edu

Daryl Cooper

Department of Mathematics

University of California

Santa Barbara, CA 93106-3080 cooper@math.ucsb.edu

Jiang-Hua $\mathrm{Lu}$

Department of Mathematics

The University of Hong Kong

Pokfulam Rd., Hong Kong jhlu@maths.hku.hk
Don Blasius

Department of Mathematics University of California

Los Angeles, CA 90095-1555

blasius@math.ucla.edu

Robert Finn

Department of Mathematics Stanford University

Stanford, CA 94305-2125

finn@math.stanford.edu

Sorin Popa

Department of Mathematics

University of California

Los Angeles, CA 90095-1555

popa@math.ucla.edu

Paul Yang

Department of Mathematics

Princeton University

Princeton NJ 08544-1000

yang@math.princeton.edu

\section{PRODUCTION}

Silvio Levy, Scientific Editor, production@msp.org

\section{SUPPORTING INSTITUTIONS}

ACADEMIA SINICA, TAIPEI

CALIFORNIA INST. OF TECHNOLOGY

INST. DE MATEMÁTICA PURA E APLICADA

KEIO UNIVERSITY

MATH. SCIENCES RESEARCH INSTITUTE

NEW MEXICO STATE UNIV.

OREGON STATE UNIV.

\author{
STANFORD UNIVERSITY \\ UNIV. OF BRITISH COLUMBIA \\ UNIV. OF CALIFORNIA, BERKELEY \\ UNIV. OF CALIFORNIA, DAVIS \\ UNIV. OF CALIFORNIA, LOS ANGELES \\ UNIV. OF CALIFORNIA, RIVERSIDE \\ UNIV. OF CALIFORNIA, SAN DIEGO \\ UNIV. OF CALIF., SANTA BARBARA
}

\author{
Vyjayanthi Chari \\ Department of Mathematics \\ University of California \\ Riverside, CA 92521-0135 \\ chari@math.ucr.edu \\ Kefeng Liu \\ Department of Mathematics \\ University of California \\ Los Angeles, CA 90095-1555 \\ liu@math.ucla.edu \\ Jie Qing \\ Department of Mathematics \\ University of California \\ Santa Cruz, CA 95064 \\ qing@cats.ucsc.edu
}

These supporting institutions contribute to the cost of publication of this Journal, but they are not owners or publishers and have no responsibility for its contents or policies.

See inside back cover or msp.org/pjm for submission instructions.

The subscription price for 2013 is US \$400/year for the electronic version, and \$485/year for print and electronic.

Subscriptions, requests for back issues and changes of subscribers address should be sent to Pacific Journal of Mathematics, P.O. Box 4163, Berkeley, CA 94704-0163, U.S.A. The Pacific Journal of Mathematics is indexed by Mathematical Reviews, Zentralblatt MATH, PASCAL CNRS Index, Referativnyi Zhurnal, Current Mathematical Publications and the Science Citation Index.

The Pacific Journal of Mathematics (ISSN 0030-8730) at the University of California, c/o Department of Mathematics, 798 Evans Hall \#3840, Berkeley, CA 94720-3840, is published monthly except July and August. Periodical rate postage paid at Berkeley, CA 94704, and additional mailing offices. POSTMASTER: send address changes to Pacific Journal of Mathematics, P.O. Box 4163, Berkeley, CA 94704-0163.

PJM peer review and production are managed by EditFLOW ${ }^{\circledR}$ from Mathematical Sciences Publishers.

PUBLISHED BY

mathematical sciences publishers

nonprofit scientific publishing

http://msp.org/

(C) 2013 Mathematical Sciences Publishers 


\section{PACIFIC JOURNAL OF MATHEMATICS}

Volume $263 \quad$ No. $1 \quad$ May 2013

Biharmonic hypersurfaces in complete Riemannian manifolds

Luis J. Alías, S. Carolina García-Martínez and Marco

RIGOLI

Half-commutative orthogonal Hopf algebras

JULIEN BiCHON and MiCHEL DUBOIS-ViOLETTE

Superdistributions, analytic and algebraic super Harish-Chandra pairs

Claudio CARMELI and RITA FIORESI

Orbifolds with signature $\left(0 ; k, k^{n-1}, k^{n}, k^{n}\right)$

Angel Carocca, Rubén A. Hidalgo and Rubí E.

RODRÍGUEZ

Explicit isogeny theorems for Drinfeld modules

IMIN CHEN and YOONJIN LEE

Topological pressures for $\epsilon$-stable and stable sets

XiAnfeng Ma and ERCAi Chen

Lipschitz and bilipschitz maps on Carnot groups

WILLIAM MEYERSON

Geometric inequalities in Carnot groups

FRANCESCOPAOLO MONTEFALCONE

Fixed points of endomorphisms of virtually free groups

PEDRO V. SILVA

The sharp lower bound for the first positive eigenvalue of the

Folland-Stein operator on a closed pseudohermitian $(2 n+1)$-manifold

CHIN-TUNG Wu

Remark on "Maximal functions on the unit $n$-sphere" by Peter M. Knopf 253 (1987)

HONG-QUAN LI 